\title{
Les pancréas bioartificiels
}

En " concurrence " avec la greffe d'îlots de Langerhans, le pancréas bioartificiel constitue l'une des voies possibles du traitement idéal des diabétiques : l'administration d'insuline régulée en permanence par la glycémie.

\section{Gérard Reach}

Maître de recherches Inserm

Michel Y. Jaffrin

Professeur à l'Université de technologie de Compiègne

\section{ADRESSE}

G. Reach : Inserm U 290, Hôpital Saint-iazare, 107, rue du Faubourg-Saint-Denis, 75010 Paris. M.Y. Jaff rin : Université de technologie de Compiègne, Cnrs UA 858, département Génie Biologique, BP 233, 60206 Compiègne. e développement d'organes artificiels pour pallier la défaillance de grandes fonctions de l'organisme constitue un des domaines-clef du génie biomédical : dans tous les cas, ce développement passe par l'analyse des fonctions de l'organe défaillant, le choix de la (ou des) fonctions(s) qu'il est essentiel de suppléer, enfin l'élaboration d'un moyen de reproduire cette fonction par un appareil artificiel : hémodialyseurs, pompes cardiaques artificielles, oxygénateurs, articulations artificielles constituent autant d'applications de cette démarche.

Le concept d'organes " bioartificiels ou hybrides " procède d'une approche différente : ici, on utilisera le tissu vivant lui-même, avec la plénitude de ses fonctions, et la partie artificielle de l'appareil aura pour rôle de protéger ce tissu contre le rejet immun, en le séparant physiquement du receveur par une membrane. Un tel principe peut donc s'appliquer au remplacement de tous les tissus dont la fonction ne nécessite pas un contact direct avec l'hôte (fonction de sécrétion ou de détoxication, par exemple).

Le but de cet article est de présenter les questions posées par le développement de pancréas bioartificiels, comme champ d'application privilégié de ce nouveau type d'organes artificiels.

\section{Pourquoi un pancréas bioartificiel?}

Le diabète sucré insulinodépendant est une affection qui touche environ I 20000 sujets en France. Il est caractérisé par une perte de la capacité du pancréas à sécréter l'insuline, une hormone qui joue un rôle essentiel dans la fonction de nutri- tion et dans l'homéostasie glycémique. Le traitement actuel, qui vise à remplacer l'insulinosécrétion défaillante par l'administration exogène d'insuline (par injections discontinues ou par perfusion continue au moyen d'une pompe), est imparfait : en effet, à la différence de l'insulinosécrétion physiologique, cette administration d'insuline n'est pas régulée en permanence par la glycémie concomitante : il est donc difficile d'éviter la survenue de pics hyperglycémiques ou d'épisodes hypoglycémiques, même au prix d'une adaptation fréquente des doses d'insuline en fonction des résultats de mesures glycémiques et de la recherche de sucre dans l'urine. A court terme, les accidents hypoglycémiques peuvent représenter une entrave à une vie sociale et professionnelle normale; à long terme, l'hyperglycémie chronique est au moins en partie responsable de la survenue de complications sévères, en particulier rénales et oculaires [I].

On comprend que le développement de systèmes d'administration d'insuline "en boucle fermée ", dans lesquels l'insuline serait délivrée d'une manière automatiquement régulée par la glycémie, constituerait un progrès décisif dans le traitement du diabète sucré. Il s'agit d'un exemple typique d'" organe artificiel " selon la définition donnée plus haut : la fonction à remplacer est ici une administration d'insuline asservie par la glycémie. Cet objectif peut être réalisé de manière entièrement artificielle : par exemple, les " pancréas artificiels électromécaniques ", développés dans les années soixante-dix, associent un capteur de glucose qui permet une mesure glycémique continue générant un signal électrique, et un ordi- 
nateur qui traite ce signal et commande le débit d'une pompe [2]; la faible longévité du capteur de glucose (au mieux quelques jours) limite actuellement le développement de ces systèmes pour une application clinique. On essaie également de développer un " pancréas artificiel chimique ", dans lequel l'insuline, fixée à un support, serait libérée lorsque la glycémie s'élève : il est possible de montrer, du moins in vitro, que la liaison de certaines insulines glycosylées aux récepteurs des oses des lectines peut être déplacée par le glucose [3].

L'alternative biologique, utilisant le tissu pancréatique vivant (transplantation de pancréas ou d'îlots de Langerhans isolés), se heurte actuellement à deux obstacles : le rejet immun du greffon et la difficulté d'approvisionnement en tissu transplantable. C'est dire l'intérêt qu'aurait le développement d'un pancréas bioartificiel (figure I) qui permettrait l'utilisation de tissu pancréatique hétérologue, voire allogénique. En effet, il s'agit là d'une application parfaite du concept d'organes bioartificiels puisque la fonction que l'on souhaite suppléer (la sécrétion d'insuline régulée en fonction de la glycémie) ne nécessite pas un con- tact physique avec l'hôte : le poids moléculaire du glucose (I 80 ) et de l'insuline (5 900) permet d'envisager l'utilisation de membranes semiperméables qui seraient imperméables aux lymphocytes et aux immunoglobulines.

\section{2 types de pancréas bioartificiels}

Plusieurs solutions géométriques sont possibles pour concrétiser sous forme d'un outil thérapeutique le principe de pancréas bioartificiel ( figure I). On peut les classer schématiquement en deux groupes [4]. Dans les pancréas bioartificiels extravasculaires, le tissu pancréatique est placé à l'intérieur d'une chambre dont les parois sont constituées par la membrane : il s'agit de chambres de diffusion (un anneau sur lequel sont fixées deux rondelles de membrane), de microcapsules, ou de fibres creuses, à l'intérieur desquelles se trouvent les îlots de Langerhans; ils pourraient être implantés dans la cavité péritonéale. Les pancréas bioartificiels vasculaires sont conçus sous forme d'un canal dont la paroi est constituée par la membrane et à l'intérieur duquel circule le sang de l'hôte diabétique, les îlots de Langerhans étant placés de l'autre côté de la membrane; ils seraient donc connectés-à un axe vasculaire, tel un shunt artério-veineux.

Quelle que soit la géométrie envisagée, la conception d'un pancréas bioartificiel doit respecter plusieurs impératifs [5]; les deux premiers découlent de la définition même du pancréas bioartificiel : d'abord la membrane doit assurer une protection efficace contre le rejet immun et ensuite le pancréas bioartificiel doit fonctionner comme un système d'administration d'insuline en boucle fermée, c'est-à-dire répondre à une charge en glucose par une augmentation de sa production d'insuline; cette réponse doit être suffisamment rapide pour permettre une régulation efficace de la glycémie. Les deux impératifs suivants ont trait au caractère potentiellement implantable à long terme de la prothèse : les îlots de Langerhans placés à l'intérieur de l'appareil doivent avoir une survie fonctionnelle prolongée et les propriétés fonctionnelles d'échanges et d'immunoprotection de la membrane ne doivent pas être altérées par un contact prolongé avec les tissus de l'hôte; dans le cas des systèmes vasculaires, il faudra de plus éviter la thrombose

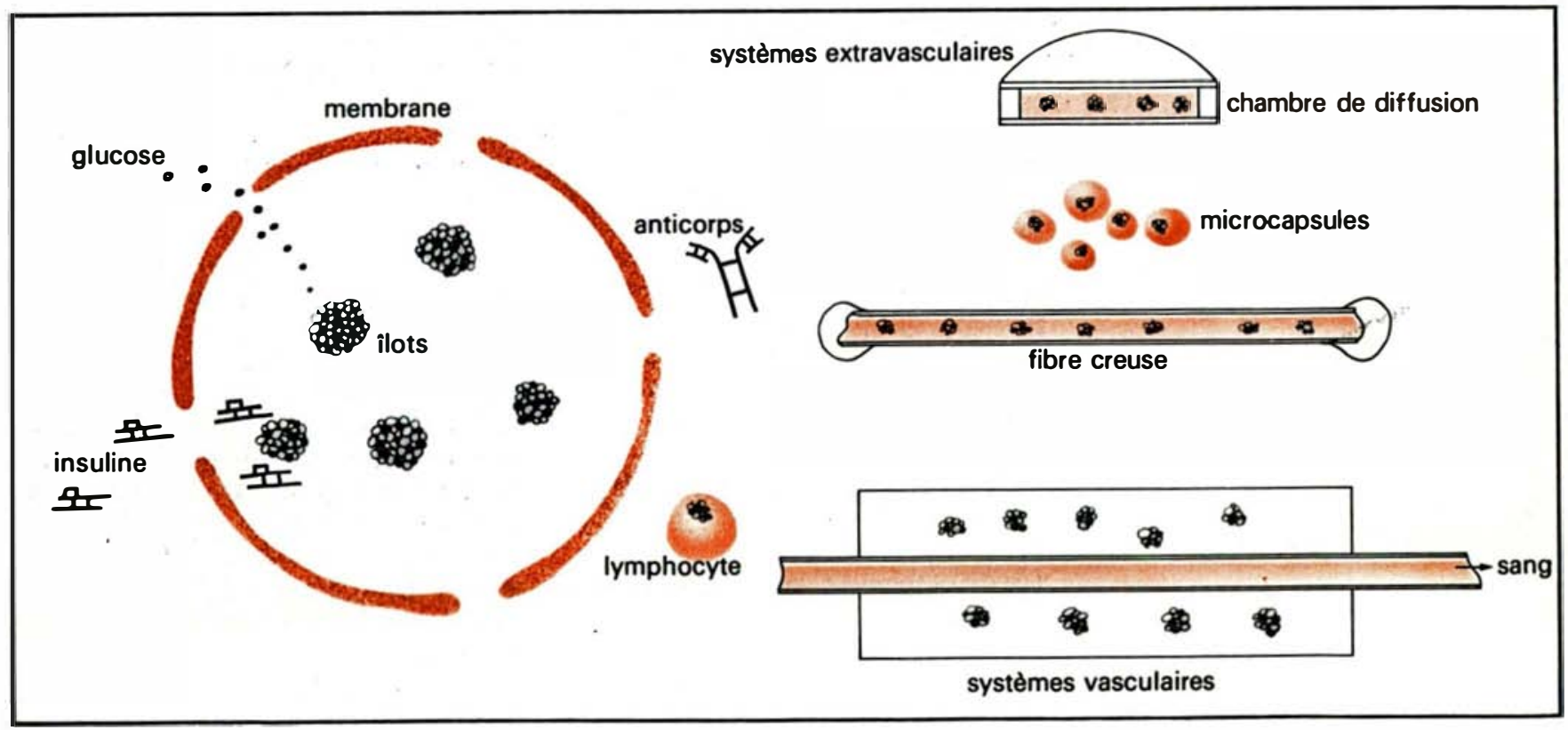

Figure 1. A gauche : principe des pancréas bioartificiels. A droite : différents types de pancréas bioartificiels. 
du canal sanguin. Enfin, la conception du pancréas bioartificiel doit tenir compte du nombre d'îlots de Langerhans nécessaire à la correction du diabète sucré humain (que l'on peut estimer à 3000 îlots par kilogramme, d'après des expériences de transplantation, soit environ 200000 îlots chez l'homme).

\section{Quel type de membrane?}

Les membranes artificielles peuvent être subdivisées en deux types : les membranes microporeuses, utilisées par-exemple pour la plasmaphérèse, sont imperméables aux cellules, mais perméables aux protéines de gros poids moléculaire comme le complément et les immunoglobulines; les membranes semi-perméables, utilisées en hémodialyse, ne sont pas perméables aux protéines d'un poids moléculaire supérieur à 50000 comme l'albumine. On a proposé d'utiliser les deux types de membrane, mais ceci mérite d'être discuté : s'il s'agissait seulement d'éviter la survenuc du rejet immun du tissu transplanté, il est vraisemblable qu'une immunoprotection efficace pourrait être assurée par des membranes microporeuses : en effet, des cellules allogéniques placées dans des chambres de diffusion utilisant des membranes Nucléopore (taille des pores $0,45 \mu \mathrm{M})$, qui empêchent seulement le contact cellulaire entre le greffon et le receveur, peuvent être transplantées avec succès [6]. Ainsi, la transplantation chez le rat rendu diabétique par la streptozotocine, d'îlots de Langerhans hétérologues placés dans de telles chambres n'entraîne pas chez le receveur l'apparition d'anticorps anti-îlots [7]. En fait, la situation est plus complexe dans le cas spécifique de la transplantation d'îlots de Langerhans chez l'homme diabétique, pour deux raisons : d'abord le sérum de sujets humains normaux et diabétiques contient des facteurs cytotoxiques pour les îlots de Langerhans; ces facteurs ne sont pas dialysables, sont détruits par la chaleur ou le traitement du sérum par le zymosan- $A$, ce qui suggère une liaison avec le système complémentaire [8]. Ensuite, il existe spontanément chez $m / s n^{\circ} 2$ iol. 2 fërrier 86 de nombreux patients atteints de diabète sucré insulinodépendant des anticorps cytotoxiques en présence de complément pour les îlots de Langerhans, ce qui témoigne de la nature auto-immune du diabète sucré humain [9]; bien que l'effet de ces anticorps n'ait été démontré qu'in vitro et que leur rôle pathogène réel reste incertain, il paraît hasardeux d'utiliser pour une implantation à long terme une membrane qui serait perméable aux anticorps et au complément : de fait, des cellules placées dans des chambres de diffusion sont rejetées lorsque les animaux receveurs ont été au préalable immunisés contre ces cellules [10]. Il semble donc nécessaire, lors du développement d'un pancréas bioartificiel, de s'assurer du pouvoir d'exclusion moléculaire de la membrane que l'on souhaite utiliser. L'imperméabilité aux immunoglobulines et au complément a été démontrée, notamment dans le cas des microcapsules d'alginate-polylysine, qui ont été proposées pour la conception d'un pancréas bioartificiel [II] : des cellules insulinosécrétrices microencapsulées sont protégées (figure 2) contre l'effet cytotoxique de sérums de sujets diabétiques [12].

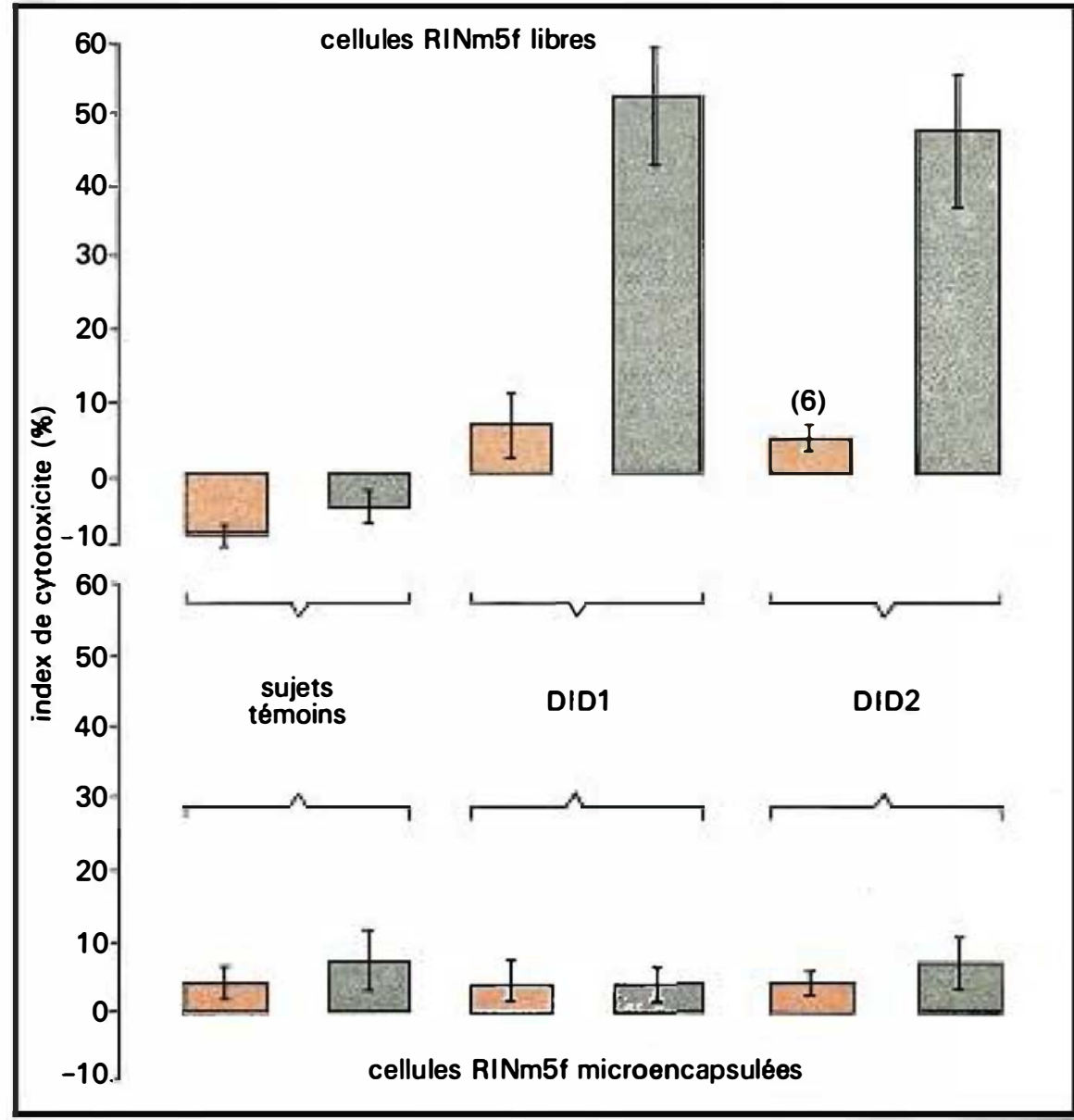

Figure 2. Démonstration de l'immunoprotection par la microencapsulation. Des cellules RINm5f (une lignée cellulaire insulinosécrétrice), libres (en haut) ou microencapsulées (en bas) sont incubées en présence de sérum de sujets témoins et de deux patients diabétiques (DIDI et $\left.D I D_{2}\right)$, et de complément natif (barres grises) ou inactivé par la chaleur (barres roses). Les cellules ont été au préalable marquées avec $d u$ si Chrome, et on détermine en fin d'incubation le pourcentage de chromi libéré dans le milieu, ce qui permet de calculer un index de cytotoxicité. Il apparaî clairement que les cellules sont tuées à l'état libre par le sérum des sujets diabétiques et qu'elles sont protégées contre cet effet cytotoxique lorsqu'elles sont microencapsulées. D'après [12], modifié. 


\section{REFÉRENCES}

I. Tchobroutsky G. Relation of diabetic control to development of microvascular complications. Diabetologia 1978; 1 5: 143-52.

2. Albisser AM, Leibel BS, Ewart G, Davidovac Z, Botz CK, Zingg W. An artificial endocrine pancreas. Diabetes 1974; 23: 389-96.

3. Brownlee M, Cerami A. Glycosylated insulin complexed to concanavalin A: a biochemical basis for a closed-loop insulin delivery system. Diabetes 1983; 32: 499-504

4. Scharp DW, Mason NS, Sparks RE. Islet immuno-isolation: the use of hybrid artificial organs to prevent islet tissue rejection. World 7 Surg 1984; 8: 221-9.

5. Reach G. Bioartificial pancreas. Present state and future prospects. Biomed Biochem Acta 1984; 5: 569-76.

6. Algire GH, Weaver JM, Prehn RT. Growth of cells in vivo in diffusion chambers. I. Survival of homografts in immunized mice. $7 \mathrm{Nat}$ Cancer Inst 1954; 15: 493-501.

7. Theodorou NA, Easterbrook $P$, Tyhurst $M$, Howell SL. Islets of Langerhans implanted in diffusion chambers do not initiate antibody production. Transplantation 1981; 31: 89-90.

8. Lernmark A, Sehlin J, Täljedahl IB, Kromann $H$, Nerup J. Possible toxic effects of normal and diabetic patient serum on pancreatic B-cells Diabetologia 1978; 14: 25-31.

9. Assan R. Diabète sucré et autoimmunité Médecine/Sciences 1985; 1 : 178-85.

10. Weaver JM, Algire GH, Prehn RT. The growth of cells in vivo in diffusion chambers. II. The role of cells in the destruction of homografts in mice. 7 Nat Cancer Inst 1955; 15: 1737-57.

I I. Lim F, Sun A. Microencapsulated islets a bioartificial endocrine pancreas. Science 1980; 210: 908-10.

12. Darquy S, Reach G. Immunoisolation of pancreatic $B$ cells by microencapsulation: an in vitro study. Diabetologia 1985; 28:776-80.

13. O'Shea GM, Goosen MFA, Sun AM. Prolonged survival of transplanted islets of Langerhans encapsulated in a biocompatible membrane. Biochim Biophys Acta 1984; 804: 133-6.

14. Altman J J, Houlbert D, Chollier A, Leduc A McMillan P, Galletti PM. Encapsulated human islet transplants in diabetic rats. Trans $\mathrm{Am} \mathrm{Soc}$ Artif Intern Organs 1984; 30: 382-6.

15. Sorensen J, Colton CK, Hillman RS, Soeldner JS. Use of a physiological pharmcokinetic model of glucosc homeostasis for assessment of performance requirements for improved insulin therapy. Diabetes Care 1982; 5: 148-56.

16. Kraegen EW, Chisholm DJ, MacNamara ME. Timing of insulin delivery with meals. Horm Metab Res 1981; 13: 365-9.
Ces données soulignent l'importance du choix du modèle expérimental pour la démonstration de l'efficacité de l'immunoprotection: le succès de la transplantation à long terme d'îlots de Langerhans hétérologues placés dans un pancréas bioartificiel ne sera pleinement démonstratif que si un modèle autoimmun est utilisé; à ce jour, le succès de l'implantation d'îlots de Langerhans microencapsulés [13] ou placés dans des fibres creuses [14] n'a été démontré que dans des modèles toxiques de diabète expérimental.

\section{Cinétique de la réponse}

Normalement, la réponse insulinosécrétrice du pancréas à une charge en glucose est rapide; on peut démontrer, soit par des techniques de modélisation cinétique [15], soit par l'utilisation chez le sujet diabétique d'un pancréas artificiel électromécanique [I6], que le temps de réponse doit être inférieur à quinze minutes pour être compatible avec la réalisation d'une administration d'insuline "en boucle fermée ". Dans le cas d'un pancréas bioartificiel, la présence de la membrane, en retardant la transmission du signal glycémique du sang aux îlots et le retour de l'insuline sécrétée, risque de gêner la réalisation de cet impératif cinétique. De fait, la diffusion du glucose et surtout de l'insuline à travers la membrane est un phénomène lent [17], ce qui explique la lenteur (plus de vingt minutes) de la réponse à une charge en glucose de pancréas bioartificiels de type vasculaire dont la fonction était évaluée in vitro $[18,19]$.

Cet obstacle peut être surmonté de deux façons. D'abord en diminuant au maximum le volume du compartiment contenant les îlots, afin de limiter l'effet de dilution [18] : il est donc vraisemblable que parmi les pancréas bioartificiels extra-vasculaires, les microcapsules et les fibres creuses représenteraient une géométrie plus favorable que les chambres de diffusion; toutefois, la cinétique dc réponse de ces systèmes n'a pas été évaluée à ce jour, par exemple en les implantant chez des animaux diabé- tiques totalement insulinopéniques et en dosant l'insulinémie au décours d'une charge en glucose. Une deuxième solution consiste à associcr aux mouvements par diffusion du glucose et de l'insuline à travers la membrane, des mouvements par convection, ce qui est possible dans les pancréas bioartificiels de type vasculaire : ceci revient à faire balayer le compartiment contenant les îlots par un flux d'ultrafiltrat produit à travers la membrane à partir du sang circulant; ce flux apporterait aux îlots le signal glycémique et remporterait l'insuline. De fait, des îlots de Langerhans périfusés in vitro avec un ultrafiltrat de sang répondent normalement au glucose, ce qui démontre la faisabilité d'une telle approche [20]. Le développement d'un tel pancréas bioartificiel pourrait utiliser l'existence du flux spontané d'ultrafiltration-réabsorption qui existe dans les systèmes de type vasculaire: on peut démontrer [2I] que la baisse de la pression hydrostatique due à l'écoulement du sang produit un flux d'ultrafiltration dans la première moitié du canal, suivi d'une réabsorption de liquide à travers la membrane (figure $3 A$ ); ceci rappelle le phénomène de Starling de la microcirculation, dans lequel une ultrafiltration au niveau de l'artériole génère du liquide interstitiel qui est réabsorbé au niveau de la veinule; une telle disposition vasculaire existe au niveau de l'îlot de Langerhans [22]. Il est possible de visualiser ce "phénomène de Starling artificiel " dans les pancréas bioartificiels de type vasculaire en faisant circuler à travers le "canal sang " une molécule colorée de faible poids moléculaire, la vitamine B I2. Si on donne au canal sang une forme en $U$ ( figure $3 B$ ), on raccourcit encore le temps de réponse puisque le flux d'ultrafiltration-réabsorption traverse désormais le compartiment contenant les îlots comme un court circuit [23]. Le temps de réponse au glucose de la production d'insuline d'un pancréas bioartificiel construit selon ce principe a été trouvé inférieur à cinq minutes (figure 4).

Ceci démontre qu'il est nécessaire d'introduire dans la conception d'un pancréas bioartificiel une étape de 


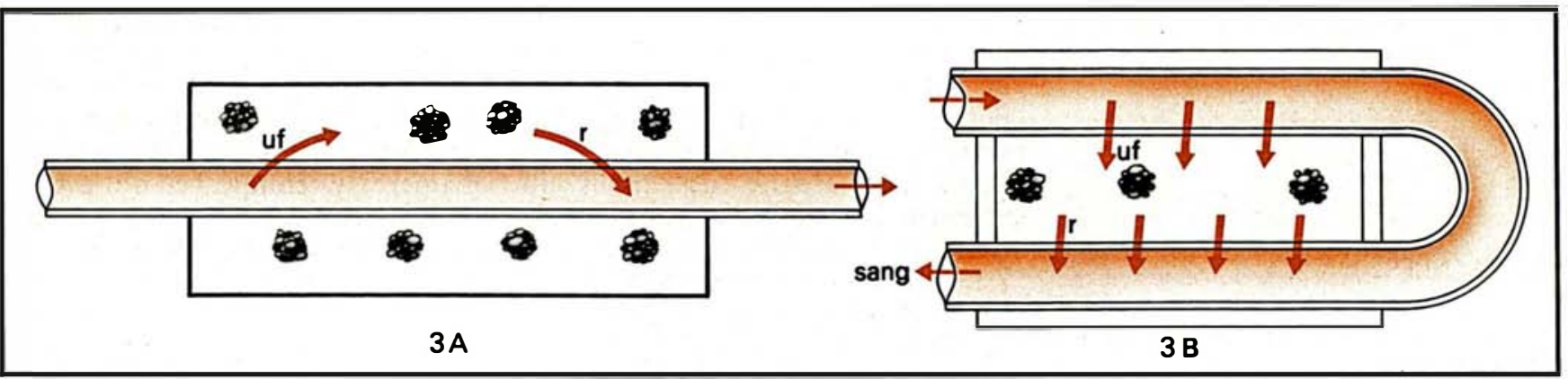

modélisation cinétique des transferts du glucose et de l'insuline à travers la membrane, de même que le développement d'un pancréas artificiel électromécanique passe par un affinement des algorithmes qui permettent de calculer la dose d'insuline administrée en fonction de la mesure de la glycémie par le capteur. Ce n'est qu'à ce prix que la géométrie d'une maquette conçue pour être évaluée chez le petit animal de laboratoire pourra être transposée sous forme d'un appareil utilisable chez l'homme.

\section{La survie des îlots immunoprotégés}

Ce troisième objectif peut être atteint : il est possible de cultiver in vitro pendant plusieurs mois des îlots de Langerhans à l'état microencapsulé [24]; de même, la survie fonctionnelle d'îlots de Langerhans placés dans un pancréas bioartificiel de type vasculaire a été démontrée lorsqu'on faisait circuler dans le canal sang un milieu de culture $[25,26]$.

A ces données de l'expérimentation in vitro (figure 5, voir p. 92) s'ajoutent les résultats de l'implantation de tissu pancréatique hétérologue à l'état microencapsulé [13] ou à l'intérieur de fibres creuses [14], chez le rat diabétique : le diabète a été corrigé pendant plus d'un an ( figure 6, voir p. 93), ce qui s'est traduit par l'absence d'apparition de cataracte ou des lésions rénales secondaires au diabète. L'évaluation in vivo de la survie d'îlots de Langerhans placés dans des pancréas bioartificiels de type vasculaire a été en général écourtćc par la survenue d'une thrombose du canal sang : la durée rapportée la plus
Figure 3. Phénomène de Starling dans les pancréas bioartificiels de type vasculaire. $3 A$ : structure linéaire; $3 B$ : structure en $U$, dans laquelle les flux d'ultrafiltration (uf) et de réabsorption ( $r$ ) traversent le compartiment contenant les ilots comme un court-circuit.

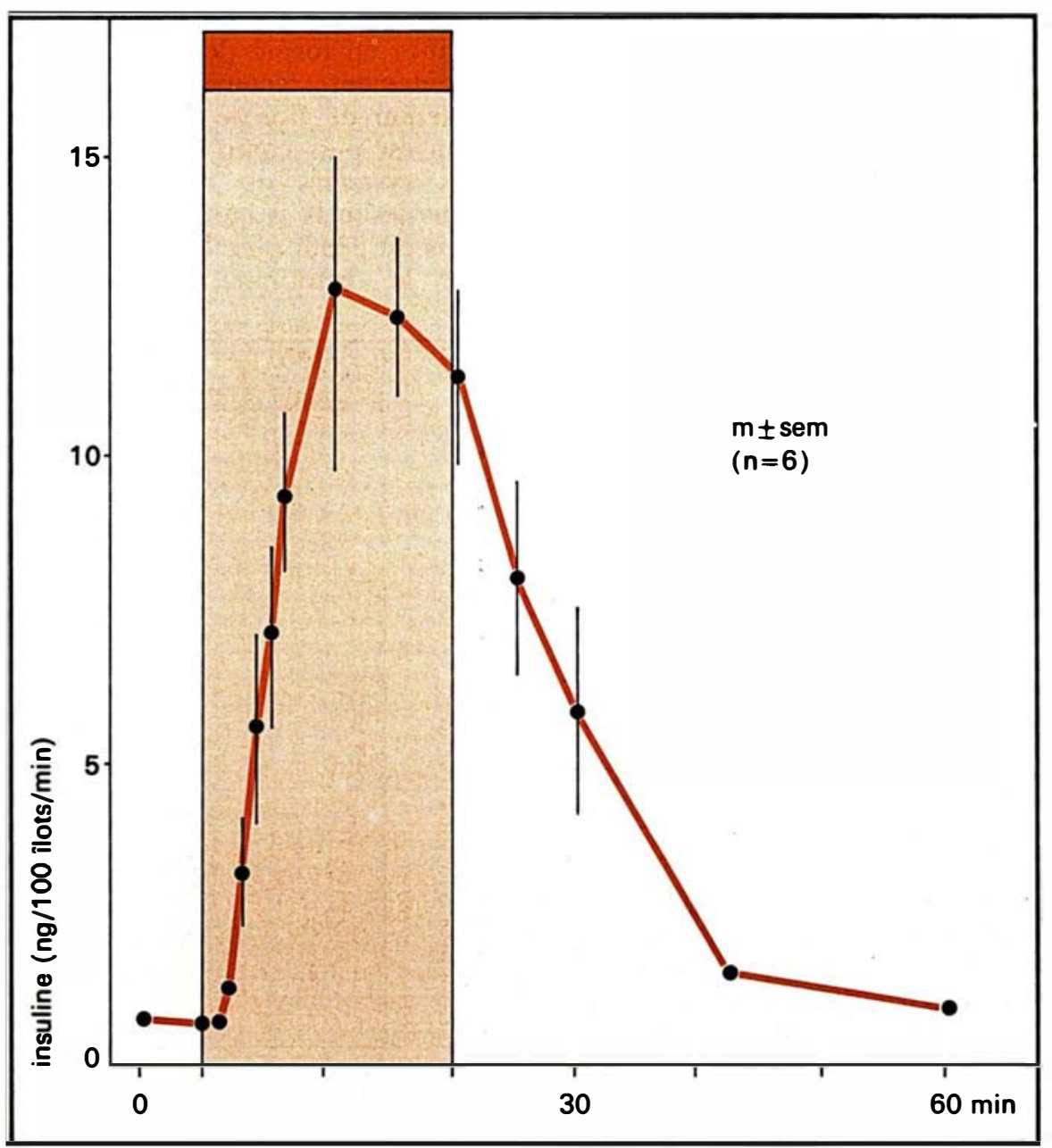

Figure 4. Réponse rapide d'un pancréas bioartifíciel au glucose. Un pancréas bioartificiel dont la structure est représentée sur la figure ${ }_{3} B$ est perfusé avec un milieu synthétique dont on augmente la concentration en glucose en onde carrée (rectangle rouge). On dose linsuline à la sortie de l'appareil. D'après [23], modifié. 


\section{REFERENCES}

17. Theodorou N, Howcll SL. An assessment of diffusion chambers for use in pancreatic islet cel transplantation. Transplantation 1979; 27: 350-3.

18. Sparks RE, Mason NS, Finley TC, Scharp DW. Development, testing and modelling of an islet transplantation chamber. Trans Am So Artif Intern Organs 1982; 28: 229-31.

19. Orsetti A, Bouhaddioui N, Crespy S, Perez R. Analyse critique de la valeur fonctionnelle d'un pancréas bio-artificiel (modèle à fibres creuses) CR Soc Biol (Paris) 1981; 175: 228-34.

20. Reach G, Poussier $P$, Sausse A, Assan R, Itoh $M$, Gerich JE. Functional evaluation of a bioartificial pancreas using isolated islets perifused with blood ultrafiltrate. Diabetes $1981 ; 30: 296$ 301 .

21. Reach G, Jaff rin MY, Vanhoutte C, Desjeux JF. Importance of convective transport in a mode of bioartificial pancreas. Am Soc Artif Intern Organs 7 1984; 7: 85:90.

22. Bonner-Weir $S$, Orci $L$. New perspectives on the microvasculature of the islets of Langerhans in the rat. Diabetes $1982 ; 31: 883-9$

23. Reach G, Jaff rin MY, Desjeux JF. A U-shaped bioartificial pancreas with rapid glucose-insulin kinetics: in vitro evaluation and kinetic modelling. Diabetes 1984; 33: 752-6r.

24. Leung YF, O'Shea GM, Goosen MFA, Sun AM. Microencapsulation of crystalline insulin or islets of Langerhans: an insulin diffusion study. Artif Organs 1983; 7: 208-12.

25. Tze WJ, Tai J, Wong FC. In vitro culture - a method of pancreatic islet preservation for transplantation. Metabolism 1980; 29: 1020-5.

26. Araki Y, Solomon BA, Basile RM, Chick WL. Biohybrid artificial pancreas. Long term insulin secretion by devices seeded with canine islets. Diabetes 1985; 34: 850-4

27. Tze W, Wong $F$, Chen L. Implantable artificial capillary unit for pancreatic islet allograft and xenograft. Diabetologia 1979; 16: 247-52.

28. Woodward SC. How fibroblasts and giant cells encapsulate implants: considerations in design of glucose sensors. Diabetes Care 1982; 5: 278-81.

29. Theodorou NA, Vrbova $H$, Tyhurst $M$ Howell SL. Problems in the use of polycarbonate diffusion chambers for syngeneic pancreatic islet transplantation in rats. Diabetologia 1980; 18: 313-7.

30. Scharp DW. Isolation and transplantation of longue est de II jours [27]. Ainsi, l'ensemble de ces données démontre de manière convaincante que des îlots de Langerhans peuvent survivre de manière prolongée lorsqu'ils sont séparés des tissus du receveur par une membrane artificielle.

La correction à long terme, vue plus haut, du diabète expérimental par des îlots microencapsulés ou placés à l'intérieur de fibres creuses, démontre que la survie fonctionnelle de la membrane de ces systèmes peut dépasser un an.

\section{Survie fonctionnelle de la membrane}

Ceci peut être dû à leur géométrie [28] : dans le cas moins favorable des chambres de diffusion, les membranes en forme de disque étaient rapidement recouvertes par une réaction de fibrose [29]. La situation est plus complexe dans le cas des systèmes de type vasculaire, dans lesquels il faut considérer, en plus du dépôt possible de protéines sur la membrane, le risque de thrombose du canal sang et des accès vasculaires. Actuellement, l'évaluation in vivo de ces pancréas bioartificiels a été réalisée chez des animaux traités par l'héparine de manière systémique, ce qui est difficilement acceptable pour une utilisation clinique à long terme chez l'homme. Le développement de tels systèmes nécessite donc une analyse soigneuse des phénomènes responsables de la survenue du phénomène de thrombose, en terme d'hémocompatibilité des biomatériaux utilisés, de la géométrie des surfaces au contact du sang, et du débit sanguin. Des progrès en matière de développement de surfaces rendues non thrombogéniques sont en particulier indispensables au développement à terme de ces projets; leur retentissement dépasserait le cadre des pancréas bioartificiels.

\section{Isolement de masse d'îlots de Langerhans}

L'isolement d'îlots de Langerhans à partir du pancréas de gros animaux ou de pancréas humains est beau-

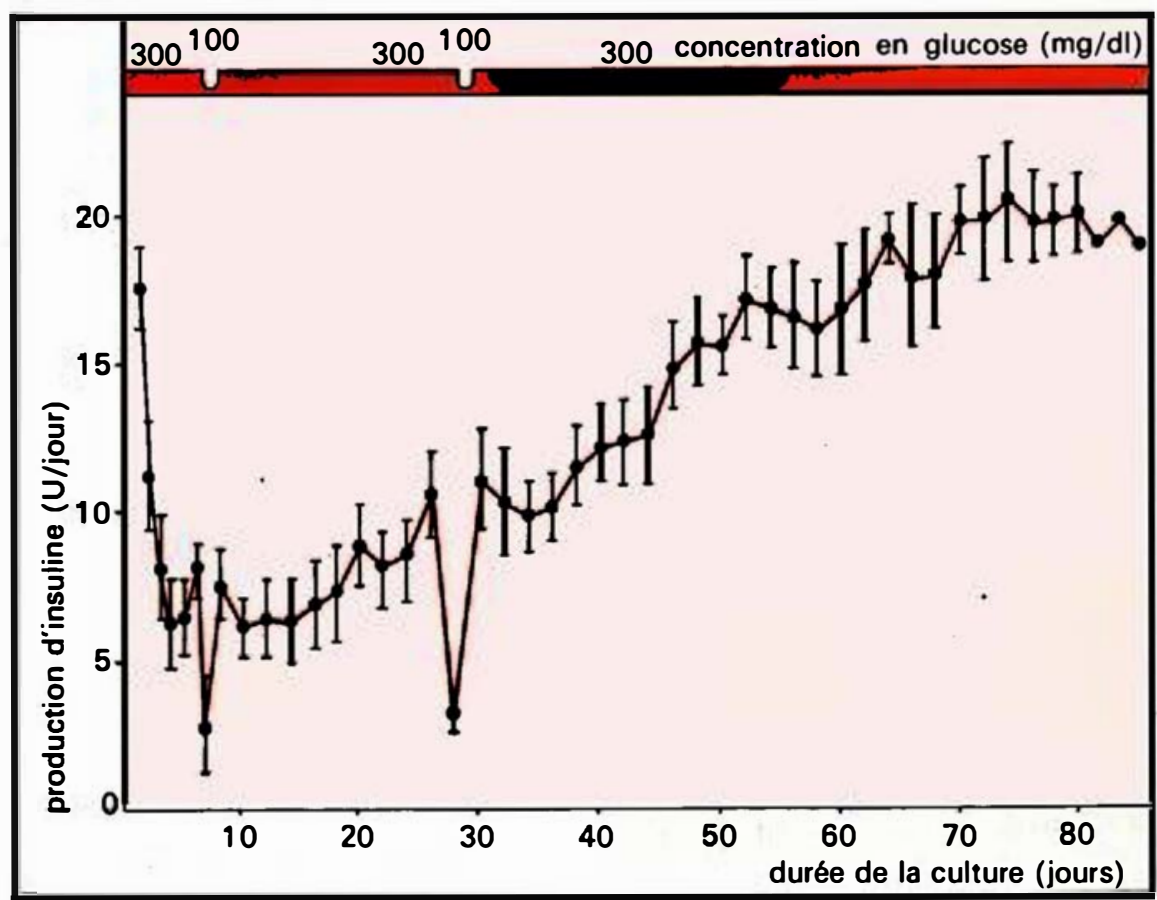

Figure 5. Survie fonctionnelle prolongée d'îlots de Langerhans placés dans un pancréas bioartificiel (expériences in vitro). Un pancréas bioartificiel, dont la structure est représentée sur la figure $3 A$, contenant 50000 ilots de chien, est perfusé pendant 80 jours avec un milieu de culture contenant des concentrations variables de glucose. L'insuline produite par jour à la sortie de l'appareil est mesurée. D'après [26], modifié. 


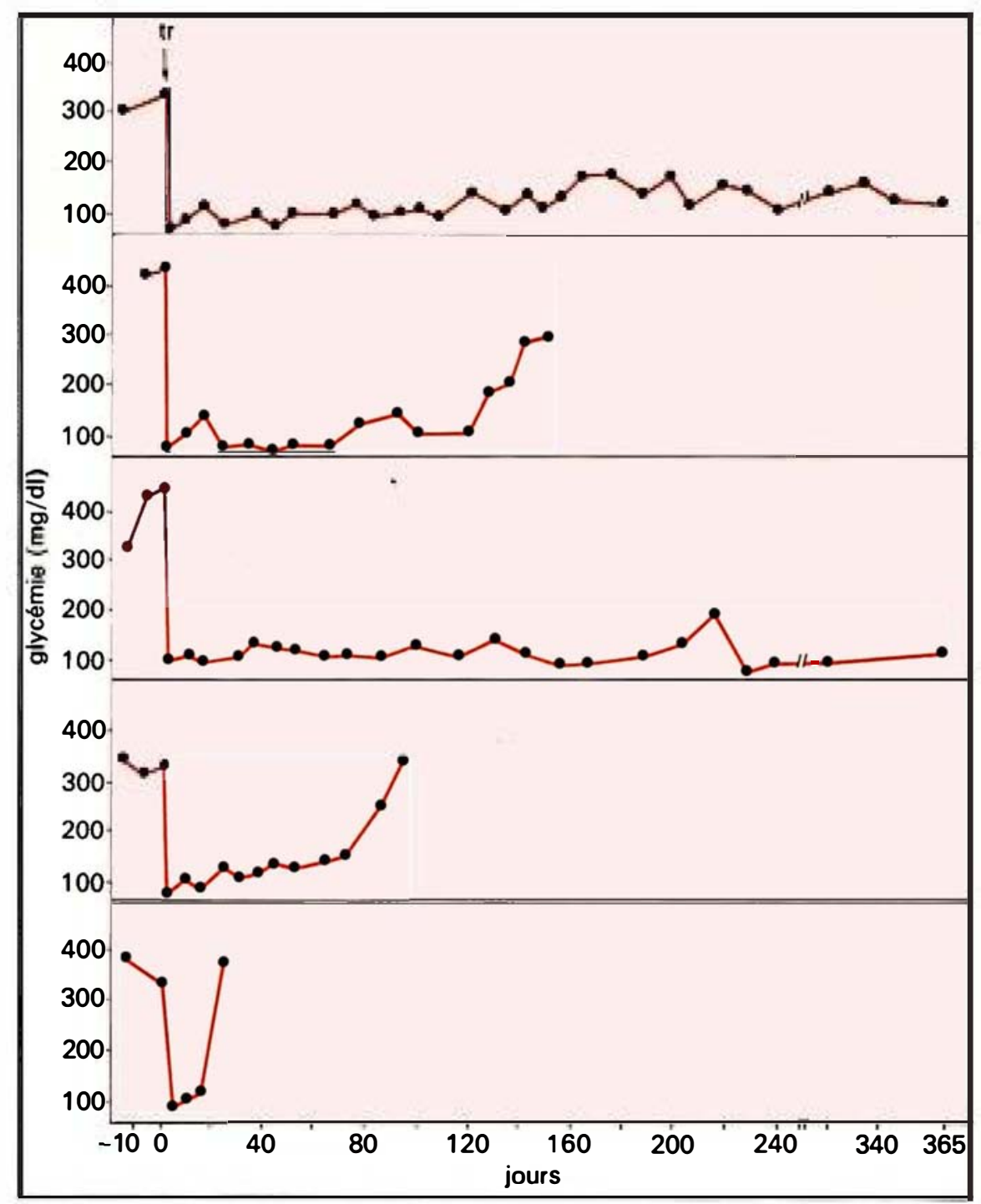

Figure 6. Normalisation à long terme de la glycémie par une unique injection (tr) de 4500 illots de Langerhans microencapsulés chez cinq rats rendus diabétiques par la streptozotocine. Chez deux animaux, le diabète a été corrigé pendant un an. D'après [I.3], modi/ié.

coup plus difficile à réaliser que l'isolement d'îlots de rongeurs, en raison du caractère plus fibreux du pancréas. Des progrès récents [30] permettent maintenant d'obtenir à partir d'un unique pancréas de bœuf, de porc, ou de chien plusieurs dizaines de milliers d'îlots de Langerhans, et certains laboratoires s'attachent à développer des méthodes d'isolement de masse sur des bases industrielles.

\section{Conclusion}

Cet article a tenté de démontrer que le concept de pancréas bioartificiel est valide puisque : (a) des îlots de
Langerhans peuvent survivre de manière prolongée alors qu'ils sont séparés des tissus du receveur par une membrane artificielle; (b) il est possible de les protéger efficacement contre le rejet immun par une membrane appropriée; (c) certains systèmes peuvent répondre avec une cinétique rapide d'insulinosécrétion à une charge en glucose; (d) il devient possible d'isoler un nombre suffisant d'îlots de Langerhans. Le développement de matériaux biocompatibles est sans doute l'étape qui permettra à ces systèmes de devenir une réalité clinique : elle nécessitera une approche multidisciplinaire

\section{Summary}

The principle of bioartificial pancreas is to permit the transplantation of non syngeneic islets of Langerhans for the treatment of human diabetes mellitus. An artificial membrane, permeable to glucose and insulin, but not so to immunoglobulins and lymphocytes, separates the islets from the host; thus, the islets would be protected against immune rejection. This review presents the evidences that I) islets of Langerhans can survive for a long period of time (up to one year) in immunoprotected state, 2) immunoprotection of endocrine cells by appropriate membranes is feasible, 3) the response time of the device to a glucose challenge can be short enough to be compatible with the achievement of closed-loop insulin delivery, 4) enough islets can be currently isolated from the pancreas of large species. The development of biocompatible materials is required to transform this attractive idea into a therapeutic tool.

\section{TIRES A PART}

Gérard Reach : Inserm U 29o, hôpital SaintLazare, 107, rue du Faubourg Saint-Denis, 75010 Paris. 